\title{
Fresh Osteochondral Allograft Transplants in the Knee: Bipolar and Beyond
}

\begin{abstract}
James P. Stannard, MD ${ }^{1,2}$ James T. Stannard, $\mathrm{PhD}^{1,2}$ Anna J. Schreiner, MD ${ }^{1,2,3}$
${ }^{1}$ Department of Orthopaedic Surgery, University of Missouri, Columbia, Missouri

${ }^{2}$ Thompson Laboratory for Regenerative Orthopaedics, University of Missouri, Columbia, Missouri

${ }^{3}$ BG Center for Trauma and Reconstructive Surgery, Eberhard Karls University of Tübingen, Tübingen, Germany

Address for correspondence James P. Stannard, MD, Department of Orthopaedic Surgery, University of Missouri, Missouri Orthopaedic Institute (4028A), 1100 Virginia Avenue, Columbia, MO 65212 (e-mail: stannardj@health.missouri.edu).

J Knee Surg 2020;33:1172-1179.
\end{abstract}

Extensive symptomatic chondral and osteochondral lesions in the knee are painful and debilitating and, left untreated, inevitably progress to posttraumatic osteoarthritis and whole-joint disease. The impact of this increasingly prevalent problem in terms of health care costs and lost productivity is enormous, with associated costs estimated at more than $\$ 200$ billion in the United States alone. ${ }^{1}$ For older and more sedentary patients, total knee arthroplasty (TKA) is commonly used to address late-stage cartilage loss such that approximately 800,000 TKAs are performed annually, with a projected $565 \%$ increase in primary TKAs by $2050 .^{2}$ However, young and active patients are not ideal candidates for TKA based on implant longevity, complications, morbidity, and risk for revision, such that treatment strategies at restoring missing hyaline cartilage and bone are highly desired for this patient population.

Fresh osteochondral allograft (OCA) transplantation has been developed as a treatment method for large $\left(>2.5 \mathrm{~cm}^{2}\right)$ focal full-thickness articular cartilage lesions, particularly of the femoral condyles. Outcomes after OCA transplantation in the knee have been moderately successful with between 71 and $85 \%$ ten-year survivorship and approximately $74 \%$ received

April 21, 2020

accepted

June 8, 2020

published online

July 20, 2020
Copyright (c) 2020 by Thieme Medical

Publishers, Inc., 333 Seventh Avenue, New York, NY 10001, USA. Tel: +1(212) 760-0888
Dol https://doi.org/ 10.1055/s-0040-1714366. ISSN 1538-8506. 
fifteen-year survivorship reported. ${ }^{3,4}$ Patients with more substantial lesions involving two articulating surfaces such that bipolar OCA transplantation is indicated have historically had less favorable reported 10 - to 15 -year survivorship rates between 40 and $70 \%$, meaning that between 30 and $60 \%$ of bipolar OCAs either failed or required revision. ${ }^{3,4}$ Need for revision or salvage (TKA) surgery was often the result of OCA cartilage erosion or delamination, or cartilage loss in nontransplanted areas of the knee. As such, many surgeons abandoned performing bipolar grafts due to these disappointing results.

In 2016, a novel graft preservation technique (Missouri Osteochondral Preservation System [MOPS], MTF Biologics, Edison, $\mathrm{NJ}$ ) was validated and became commercially available. It yields remarkably improved chondrocyte viability in OCAs at the time of transplantation and at least doubles their shelf life. ${ }^{5}$ This advance in OCA preservation technology in conjunction with technical improvements in surgical techniques fostered a progressive shift in the senior author's practice to provide options for young or active patients with extensive symptomatic chondral and osteochondral lesions in the knee to include multisurface and bipolar OCA transplantation.

\section{Surgical Techniques}

The technical improvements in surgical techniques that have evidence for improving OCA transplantation for treatment of extensive cartilage lesions include:

- cutting grafts thin (6-7 mm total thickness);

- changes in graft shape to shell grafts in most cases;

- creating a keel, or tab, on the grafts to allow for threedimensional fit and stability in the recipient site;

- drilling channels into the OCA bone;

- extensively irrigating the OCA bone with saline;

- soaking the OCA cancellous bone with autologous bone marrow aspirate concentrate (BMAC);

- stabilizing the grafts using bioabsorbable pins and nails; and

- prescribed and monitored patient-specific rehabilitation protocols. ${ }^{6-8}$

Patients are placed supine on a radiolucent table. A midline approach is made with either a medial or lateral parapatellar arthrotomy depending upon the articular surfaces in the knee being treated. A Jamshidi needle is then inserted into the distal femur and 45 to $120 \mathrm{~mL}$ of bone marrow aspirate (BMA) is collected. It is very important to draw the BMA slowly and move the needle to optimize cell recovery. The BMA is processed in the operating room to obtain BMAC using a commercially available validated system (Angel System, Arthrex, Inc., Naples, FL) for subsequent treatment of OCA bone for the indication of delivery of bone graft materials to an orthopaedic surgical site.

If the patient requires transplant of the medial or lateral tibial plateau, the first cuts are made there. We use a reciprocating saw under fluoroscopic guidance to make a vertical cut to approximately $7 \mathrm{~mm}$ depth at the margin of the respective hemiplateau, taking care to avoid damaging the cruciate ligaments. A good lateral fluoroscopic view is obtained and a

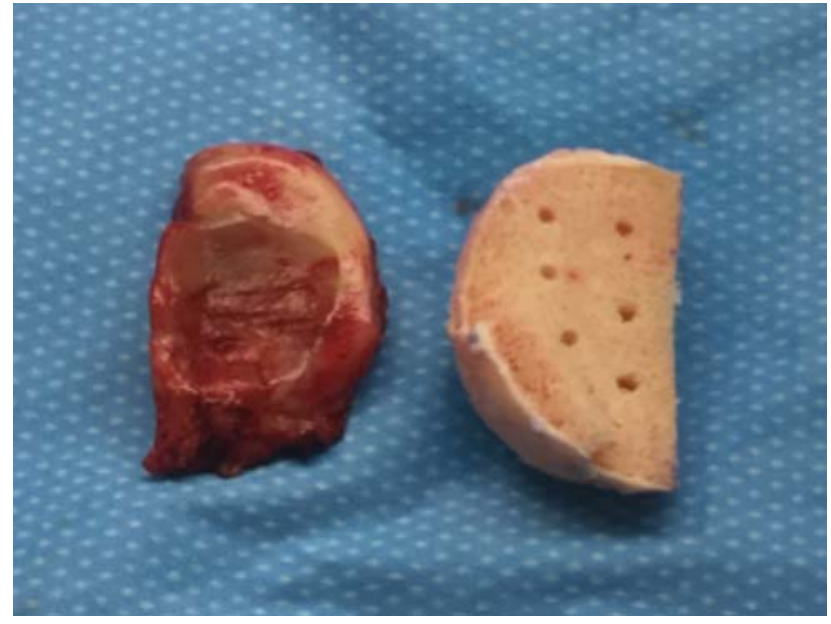

Fig. 1 Damaged tibial plateau (left) is template to cut fresh tibial plateau graft (right) with keel and drilled channels for bone marrow aspirate concentrate.

sagittal saw is used to resect 6 to $7 \mathrm{~mm}$ of tibial plateau and the associated remnant meniscus. Fluoroscopic guidance is used to match the respective tibial slope and to prevent damage to associated soft tissue and neurovascular structures. The sagittal saw is used to create a slot at the margin of the tibial recipient site to accept the keel on the OCA.

For bipolar femoral condyle-meniscotibial OCA transplants, the next step is to identify the area of articular cartilage damage on the femoral condyle. If it can be treated with a single dowel graft, we use commercially available instrumentation and methods to address the femoral lesion in that way. If the femur is not amenable to a single circular dowel graft based on size, geometry, or location, we cut a shell graft that encompasses all grossly affected articular cartilage. The sagittal saw is used to create a slot at the margin of the femoral recipient site to accept the keel on the OCA.

Custom-cut tibial plateau and femoral grafts are prepared using measurements from the recipient site and the resected tissue in conjunction with using the resected portions as templates, when possible. The grafts are made 6 to $7 \mathrm{~mm}$ thick, and both the tibial plateau and the femur have a threedimensional "keel," or tab, to allow for fit and stability in the recipient site (-Fig. $\mathbf{1}$ ).

If the patient has significant lesions of the patella and/or trochlea, we resect the entire articular surface to completely resurface it with a size-matched patella or trochlea OCA (-Fig. 2). The initial patellar resection normally leaves a distal shelf of bone and cuts a proximal slot. The trochlear resection is performed from lateral and medial trochlear ridges to create a V-shaped recipient bed. Custom-cut grafts are prepared using measurements from the recipient site and the resected tissue in conjunction with using the resected portions as templates, when possible. The grafts are made 6 to $7 \mathrm{~mm}$ thick and the patella includes a three-dimensional "keel," or tab, to allow for fit and stability in the recipient site.

When all damaged cartilage has been resected and recipient sites prepared in the patient, we begin by transplanting the femoral condyle shell allograft. We make "finishing cuts" on the graft and patient as needed to create a near-perfect fit. 


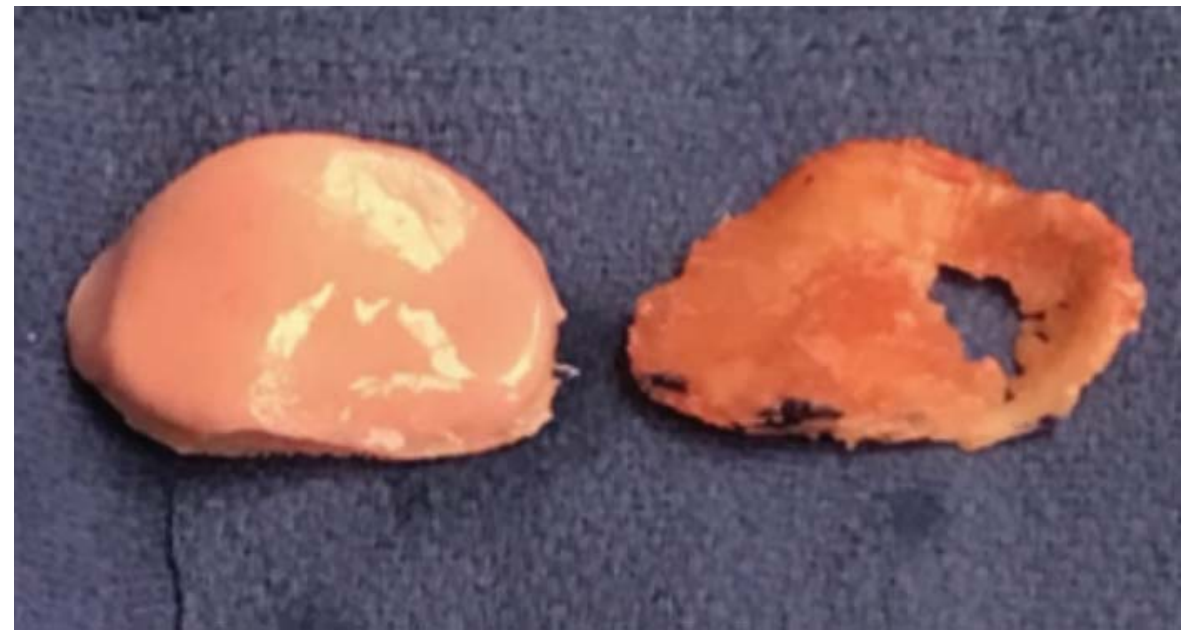

Fig. 2 Complete patella replacement graft (left) and damaged articular surface (right).

When the best fit possible has been achieved, channels are drilled into the OCA cancellous bone using a 3.2-mm drill bit. The OCA bone is thoroughly irrigated with $1 \mathrm{~L}$ of isotonic saline using a power irrigator (e.g., InterPulse, Stryker, Kalamazoo, MI). The graft bone is then saturated with the BMAC (-Fig. 3). The graft is then fixed in place using bioabsorbable nails and/or pins (e.g., Smart Nails, ConMed Utica, NY; BioPins, Arthrex, Naples, FL). This process is repeated for the tibial plateau with meniscus allograft. Great care must be used when implanting the tibial plateau to avoid damage to the meniscus allograft. In rare circumstances, we have used a femoral distractor to assist in opening the compartment to facilitate tibial plateau place-

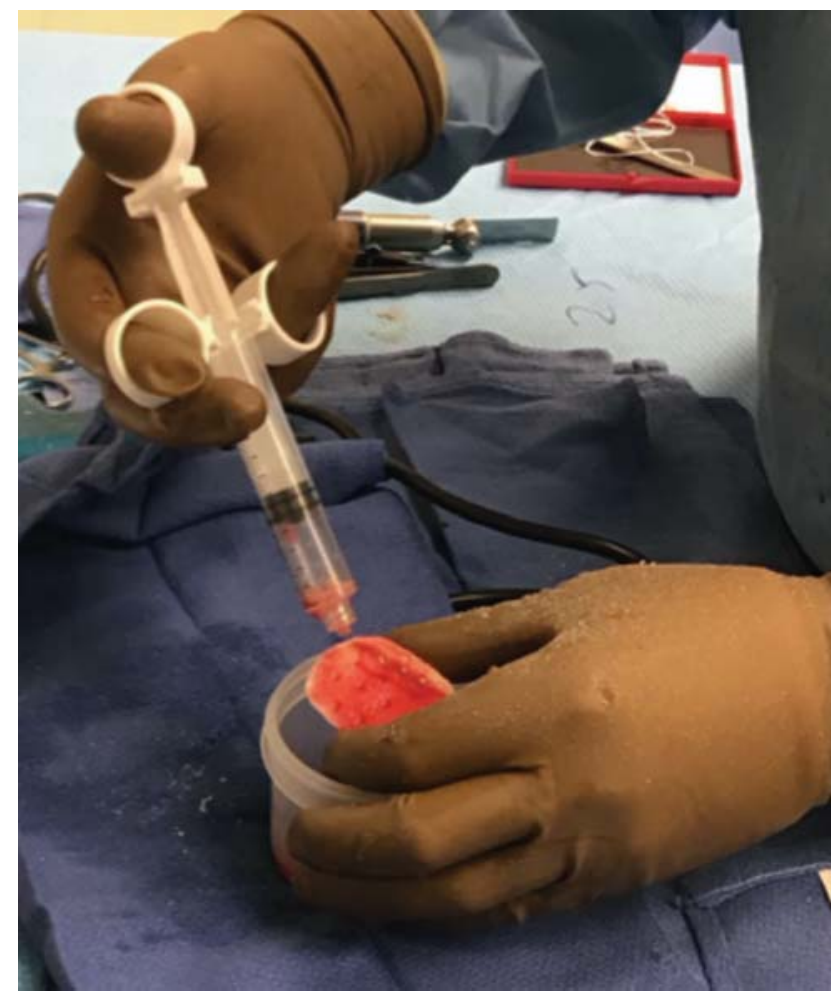

Fig. 3 Soaking patella graft with bone marrow aspirate concentrate after drilling holes. ment. The tibial graft is fixed in place using bioabsorbable nails and/or pins. The next OCA transplanted is normally the trochlea. Again, we make finishing cuts to the graft and patient to obtain the best possible fit. Channels are drilled and the OCA cancellous bone is saturated with BMAC. The graft is fixed into place using bioabsorbable nails and/or pins. Finally, the patella is resurfaced with the patellar OCA. After finishing cuts have been completed, channels have been drilled, and the OCA bone has been irrigated and then saturated with BMAC, the graft is fixed in place with bioabsorbable pins (Arthrex). Patellar tracking through range of motion is assessed and the retinaculum is repaired with suture. The meniscus allograft is attached to the patient's joint capsule using "outside-in" vertical mattress sutures of 0 Vicryl. The meniscotibial allografts we use retain the meniscotibial ligament, which we believe is crucial to success. ${ }^{7} \mathrm{We}$ then place the arthroscope into the knee and assess the meniscal stability. If necessary, we place an "all inside" suture posteriorly or "inside-out" sutures more anteriorly. It is rare that we require more than one or two arthroscopically placed sutures. The wound is then thoroughly irrigated and closed.

Our rehabilitation protocol generally involves toe touch weight bearing for 6 to 8 weeks, then $25 \%$ for 2 weeks, $50 \%$ for 2 weeks, and $75 \%$ for 2 weeks. Between the third and fourth postoperative month the patient is allowed to weight bear as tolerated and they continue to regain full motion. Radiographs are obtained at 3 months. If they look good, the patient is allowed to slowly begin use of an exercise bicycle after 4 months. At 6 months postop, if radiographs look good, they are advanced to an elliptical machine. We monitor activities carefully until 12 months postop, avoiding any impact or shearing forces. When the patient reaches 12 months and if the grafts look good, we allow the slow introduction of a single activity involving either impact or shear. ${ }^{8}$

To mitigate described complications and optimize outcomes after OCA transplantation for extensive chondral and osteochondral lesions in the knee, our institution developed a novel graft preservation system as previously described. ${ }^{5,8-13}$ Implementation of this OCA preservation technology and 
technical improvements in surgical techniques fostered a progressive shift in practice to provide options for young and active patients with extensive cartilage lesions in the knee. To effectively evaluate this progressive shift in practice in real time, all patients willing to consent to enrollment were included in a prospective registry to track, analyze, and report outcomes after OCA and meniscus allograft transplantation. With institutional review board approval and informed consent, data from patients prospectively enrolled into the registry with $\geq 1$-year follow-up data, including complications, reoperations, revisions, failures, patient-reported outcome measures (PROMs), and compliance with rehabilitation after fresh primary unipolar, multisurface, and bipolar OCA transplantations in the knee during the first 4 years of our registry have been reported. ${ }^{6,8}$

For the longest-term outcomes from our prospective registry to date, ${ }^{6} 194$ patients met inclusion criteria with $62 \%$ of cases undergoing bipolar OCA transplantation. Mean age was $37.9 \pm 12.2$ (14-69) years and mean body mass index was $28.9 \pm 5(17-46) \mathrm{kg} / \mathrm{m}^{2}$. In total, $26 \%$ of patients underwent concurrent or staged procedures in the same knee addressing comorbidities such as lower extremity malalignment or knee ligament deficiencies. Significant and clinically meaningful improvements in PROMs were noted at 3 and 4 years after OCA transplantation for this cohort of patients. When comparing PROMs between patients receiving MOPS-preserved grafts versus standard preservation (SP) grafts, mean International Knee Documentation Committee (IKDC), Single Assessment Numeric Evaluation, and Patient-Reported Outcomes Measurement Information System (PROMIS) mobility scores were significantly higher for MOPS cases at 3 years postoperatively. Initial success rates, defined as patients reporting return to functional activities with no need for revision or conversion to TKA, were $79 \%$ for all cases combined and $84 \%$ for MOPS cases. Revisions were performed in $10 \%$ of all cases and $5 \%$ of MOPS cases. Failures requiring conversion to TKA occurred in $13 \%$ of all cases and $11 \%$ of MOPS cases, with bipolar OCA transplantations being significantly more likely to fail. The majority of failures (65\%) occurred between 6 and 12 months after surgery at a mean time of 11 months. Patients who received SP grafts had been on the waiting list for MOPS grafts but had not had a sizematched graft identified using MOPS preservation when a sizematched SP graft that was less than 22 days from harvest was identified. Patients were given the option of continuing to wait for a MOPS graft or move forward with the SP graft that fit their needs. The demographics of the two groups were similar. Direct comparisons between SP and MOPS cohorts highlights the consistently superior results associated with OCA transplantation with grafts stored using the novel preservation method (-Table 1). ${ }^{6}$

Bipolar transplants were defined as involving two opposing articulating surfaces, including patellofemoral, femorotibial, and/or femoromeniscal compartments. For patients in the registry study, 58 and 64\% received bipolar OCA transplants in the SP and MOPS cohorts, respectively, with 65\% involving more than one compartment. The initial bipolar success rates were $49 \%$ for SP OCAs versus $80 \%$ for MOPS OCAs, such that MOPS bipolar cases were 4.1 times more likely than SP bipolar
Table 1 Results according to preservation method (SP $n=57$, MOPS $n=137$ )

\begin{tabular}{|l|l|l|l|}
\hline & SP & MOPS & $p$ \\
\hline Success rates & $60 \%$ & $84 \%$ & 0.028 \\
\hline Revisions & $21 \%$ & $5 \%$ & 0.0014 \\
\hline Failures & $19 \%$ & $11 \%$ & 0.048 \\
\hline VCD & $49 \%$ & $102 \%$ & $<0.05$ \\
\hline $\begin{array}{l}\text { 6-mo KM } \\
\text { survival }\end{array}$ & $98.2(89-99)$ & $98.5(94-100)$ & ns \\
\hline 1 y KM-survival & $90.8(80-97)$ & $92.0(86-96)$ & ns \\
\hline 4 y KM-survival & $77.5(65-87)$ & $89.0(82-94)$ & ns \\
\hline
\end{tabular}

Abbreviations: KM, Kaplan-Meier; MOPS, Missouri Osteochondral Preservation System; ns, no statistically significant difference; SP, standard preservation; VCD, viable chondrocyte density.

cases to be associated with successful outcomes without need for revision at $\geq 1$ year after transplantation. Failures occurred in $19 \%$ of SP cases and $15 \%$ of MOPS cases corresponding to a significantly lower failure rate for primary MOPS OCAs. ${ }^{6}$ The data presented above is for 1- to 4 -year follow-up as stated. It is highly likely that there will be some additional failures as time goes by, although most studies, and our experience, is that the overwhelming majority of failures occur within the first 18 months.

Importantly, patients who were noncompliant with the prescribed postoperative protocol during the first year after surgery were 6.7 times more likely to experience OCA transplantation failure. In fact, $46 \%$ of patients requiring TKA in the course of the study were documented to be noncompliant during the first postoperative year. These findings confirmed data from a previous study showing that compliance with procedure-specific postoperative rehabilitation protocols was associated with higher success, lower revision, and lower failure rates for patients at 1 to 3 years after osteochondral and meniscal allograft transplantation. As such, we have instituted, and highly recommend, behavioral screening, adapted patient education, and careful monitoring and counseling to positively influence this modifiable and highly relevant risk factor. ${ }^{8,14}$

\section{Results to Date}

The most recent systematic review by Melugin et $\mathrm{al}^{15}$ reported outcomes and complications for treatment of bipolar cartilage lesions of the knee including 156 OCAs in addition to cases treated using autologous chondrocyte implantation or OCA transfer. For these cases, improvement in mid-term patient-reported outcomes was noted and OCA survivorship ranged from 40 to $100 \%$. Bulk allografts and tibiofemoral compartment transplants were associated with higher failure rates. In addition, concomitant procedures were commonly performed along with OCA transplantation. A systematic review by Familiari et $\mathrm{al}^{4}$ reported patient outcomes after all types of OCA transplantation surgeries in the knee with an overall 10 -year survival rate of $78.8 \%$. 
Revision cases, patellar, and bipolar OCA transplantations were associated with worse results including a reoperation rate of $30.2 \%$ and a failure rate of $18.2 \%$. Chahal et al $^{3}$ reported a similar failure rate but a complication rate of only $2.4 \%$ with favorable outcomes and high satisfaction rates in short-term follow-up for focal and diffuse single-compartment chondral defects treated by OCA transplantation. Other reviews indicate that OCA transplantation is particularly appropriate for young and athletic patients with favorable return-to-sports rates between 75 and $82 \%{ }^{16-18}$ Importantly, bipolar OCA in the tibiofemoral compartment prolongs time to TKA and provides good overall function. ${ }^{15,19,20}$ Taken together, best current evidence suggests that the highest rates of successful OCA transplantation are observed in younger patients, unipolar lesions, normal or corrected limb alignment, and defects that are treated within 12 months of symptom onset. ${ }^{17}$ While bipolar OCA transplantation is not indicated for treatment of end-stage degenerative knee $\mathrm{OA}$, recent data supports its use for early intervention of extensive cartilage loss from other causes, especially in combination with meniscus allograft transplantation, demonstrating significant clinical improvements in cases with graft survival. ${ }^{19,21,22}$ Importantly, Cotter et al have shown that the multiplug or "snowman" technique is associated with inferior results while fresh large osteochondral shell allograft transplantation in combination with concomitant procedures allows for an anatomical restoration of osteochondral knee defects with good clinical outcomes. ${ }^{23-26}$ Prospective assessment of outcomes after OCA transplantations in the knee at our institution have recently been reported. ${ }^{6}$

In sum, the initial prospective registry data indicate that the implementation of a novel OCA preservation method that maintains high viable chondrocyte density to time of transplantation combined with modified surgical techniques and patient management protocols leads to consistently successful outcomes for unipolar, multisurface, and also bipolar OCA transplantations in the knee. This study provides further evidence that fresh OCA transplantation represents an appropriate treatment option for young and active patients with large full-thickness articular cartilage defects of the knee. ${ }^{6}$

\section{Case Example}

A 25-year-old male who was involved in a significant motor vehicle collision sustaining a severe tibial plateau crush fracture status post more than 20 surgical procedures presented for a salvage assessment. Prior to presentation, physical therapy, nonsteroidal anti-inflammatory medications, and nonweight bearing via crutches were used to offset intense pain. At presentation, physical examination, radiographic imaging, and diagnostic arthroscopic assessment revealed knee range of motion of 0-0-80 degrees, proximal tibial malunion with significant bone deformity, and extensive full-thickness posttraumatic articular cartilage lesions in all compartments of his left knee with no functional meniscal remnants (-Fig. 4). After consultation with the patient and immediate family members with comprehensive discussion of treatment options including TKA, arthrodesis, amputation, and osteochondral and meniscal allograft transplantation, he opted and provided fully informed consent for the following procedures: Left fresh MOPS-preserved OCA transplantation of the medial femoral condyle, lateral femoral condyle, trochlea, patella, medial tibial plateau, and lateral tibial plateau with arthroscopic-assisted transplant of the medial meniscus and lateral meniscus, supplemented with bone grafting of the OCA-recipient bone interfaces using autologous bone obtained from the patient's femur using a reamer irrigator aspirator. He was followed-up at 1 month, 3, 7, and 9 months, and 1 and 2 years postoperatively. Range of motion consistently improved to a flexion of 95 degrees after 1 year and 102 degrees after 2 years (-Fig. 5). Radiographs

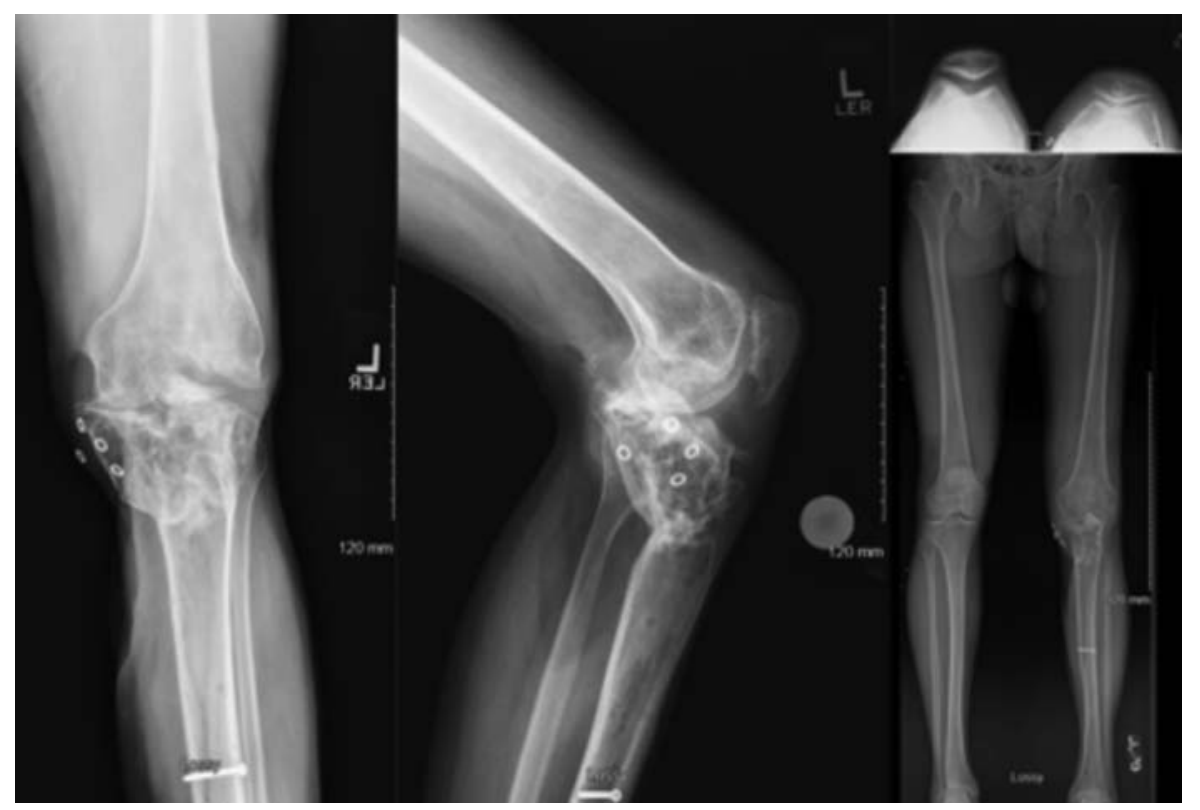

Fig. 4 Preoperative imaging of the left knee following severe tibial plateau fracture with full-thickness cartilage loss and meniscus loss from all compartments with distinctive bone deformity but adequate alignment. 


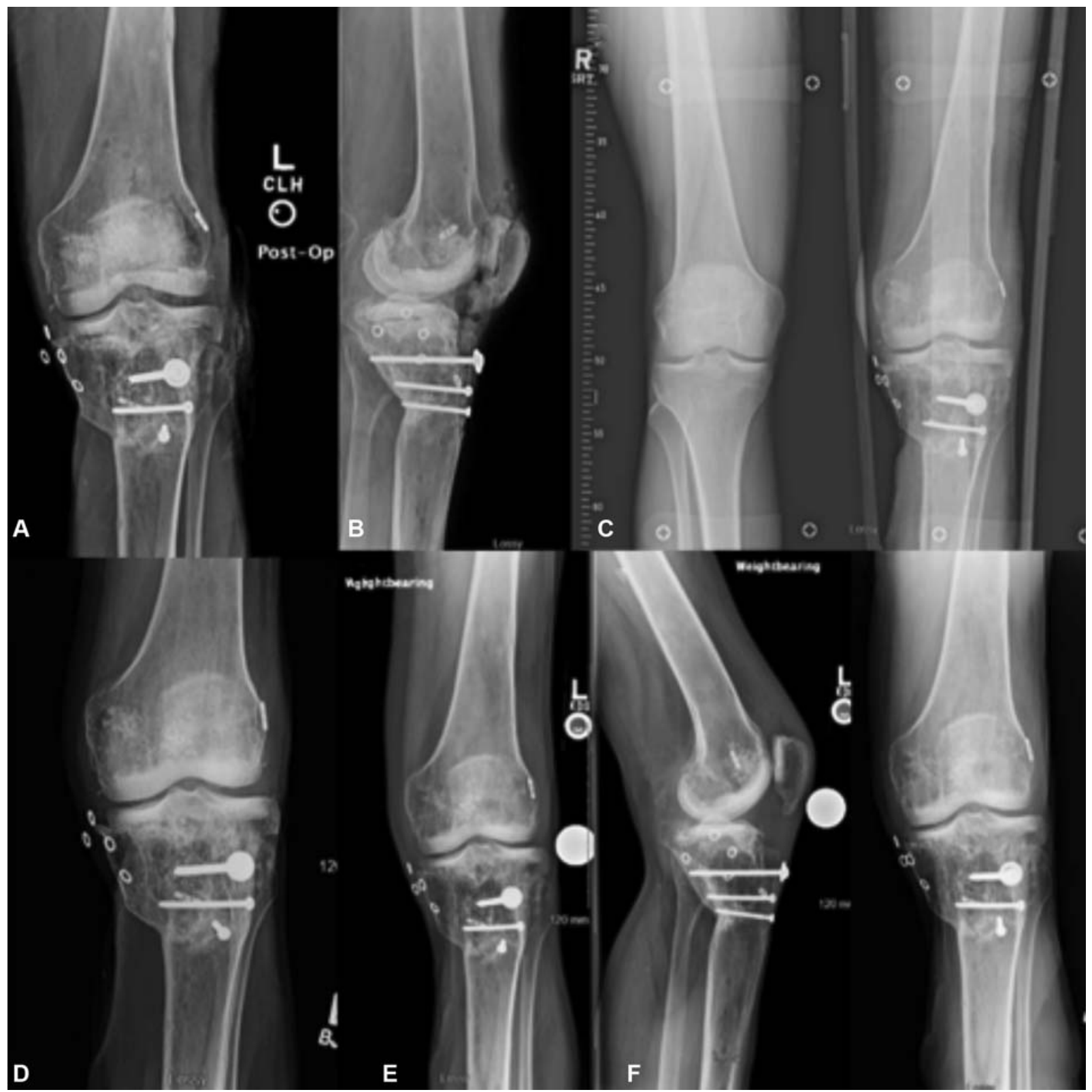

Fig. 5 Postoperative radiographs following all compartmental fresh osteochondral allograft of the left knee including both mensici: (A, B) directly postoperative anteroposterior and lateral radiographs; (C) both knees anteroposterior 1 month postoperatively; (D) anteroposterior 3 months postoperatively; (E, F) anteroposterior and lateral 7 months postoperatively; (G) anteroposterior 9 months postoperatively.

(-Fig. 6) and computed tomography (-Fig. 7) showed good graft incorporation, IKDC improved from initial 10 to 42.5 after 1 year, and pain scores decreased to 0.5 from an initial value of 7.5. The patient strictly adhered to the postoperative protocol.

\section{Future Applications}

OCA transplantation has undergone extensive basic, preclinical, and clinical research for progressive development over the last four decades and its use is steadily increasing in the United States. ${ }^{4}$ In spite of significant clinical improvements in pain and function in cases with successful graft healing, integration, and remodeling, moderate to high failure rates persist. $^{3,4,27}$ Currently, no other consistently successful treatment options are available for young or active patients with extensive symptomatic chondral and osteochondral lesions in the knee, which leads to a critical unmet need in orthopaedic health care. ${ }^{6,27,28}$ Our novel and comprehensive approach was designed to address this need based on translational research and prospective clinical assessments. As noted in our data, failures can and will occur in these complicated patients. While the number of failures appears to be decreasing compared with prior literature, they do occur and generally require either a revision of the graft transplant procedure or a conversion to a total joint arthroplasty. Both have been done successfully in our patient cohort. Further follow-up with documentation of long-term outcomes will provide insight for continued optimization for future applications for OCA transplantation, potentially including a broader spectrum of patients appropriate for 


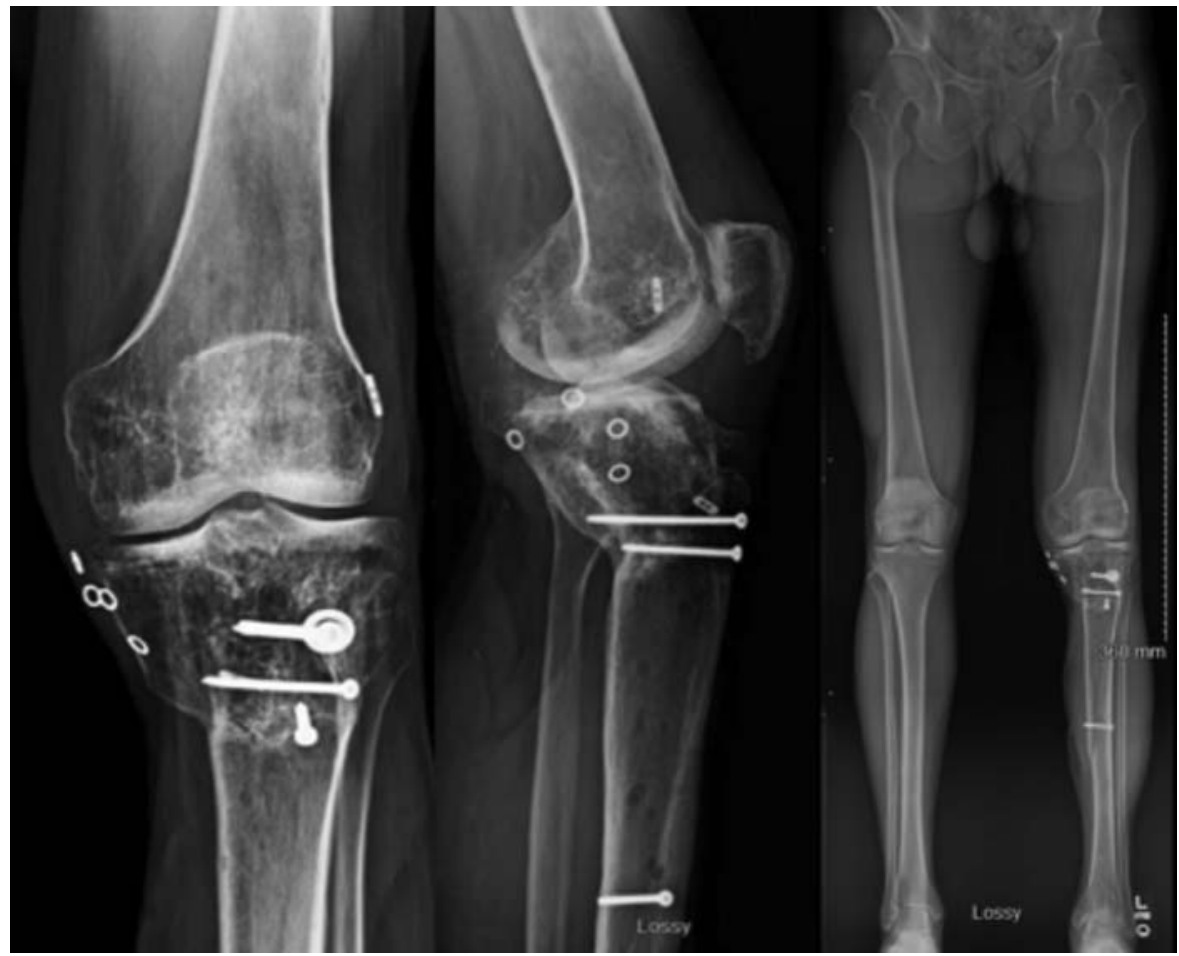

Fig. 6 One-year postoperative radiographs after partial hardware removal (prominent tubercle screw of left proximal tibia had led to associated pain).

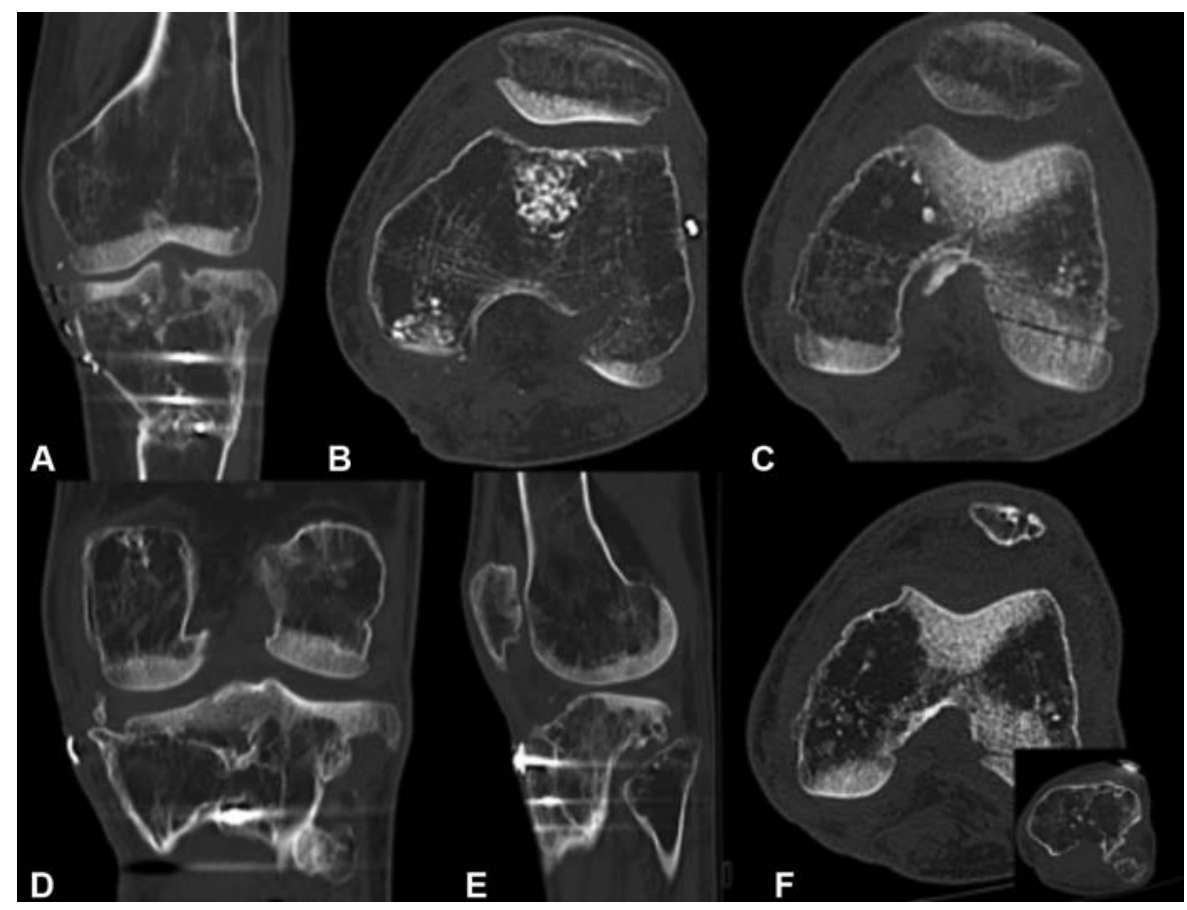

Fig. 7 (A-F) Computed tomography scans (first row -3 months and second row - 1 year postoperatively) demonstrating good graft incorporation.

this treatment. This comprehensive approach has already been successfully applied to "bipolar and beyond" OCA transplantation in patients' ankle and hip joints and has preclinical evidence for application to the upper extremity, including shoulder, elbow, and phalangeal joints. ${ }^{29,30}$ Ongoing translational research is necessary to blaze the trail in further optimizing this treatment option for patients.

\section{Authors' Contributions}

All authors have read and approved the final submitted manuscript. J. P. S., J. T. S., and A. J. S.: substantial contributions to research design, acquisition, analysis, and interpretation of data; J. P. S., J. T. S., and A. J. S.: drafting the paper and revising it critically; all authors approved the submitted and final versions. 


\section{Conflict of Interest}

J. P. S. reports grants and personal fees from Arthrex, Inc., grants from DePuy Synthes, other from Journal of Knee Surgery, grants from National Institutes of Health (NIAMS \& NICHD), personal fees and other from Thieme, grants from U.S. Department of Defense, other from AO Foundation, other from American Orthopaedic Association, other from AO North America, grants from Coulter Foundation, other from Mid-America Orthopaedic Association, personal fees from Orthopedic Designs North America, personal fees from Smith \& Nephew, outside the submitted work. All the other authors report no conflict of interest.

\section{References}

1 Vina ER, Kwoh CK. Epidemiology of osteoarthritis: literature update. Curr Opin Rheumatol 2018;30(02):160-167

2 Inacio MCS, Paxton EW, Graves SE, Namba RS, Nemes S. Projected increase in total knee arthroplasty in the United States - an alternative projection model. Osteoarthritis Cartilage 2017;25 (11):1797-1803

3 Chahal J, Gross AE, Gross C, et al. Outcomes of osteochondral allograft transplantation in the knee. Arthroscopy 2013;29(03): 575-588

4 Familiari F, Cinque ME, Chahla J, et al. Clinical outcomes and failure rates of osteochondral allograft transplantation in the knee: a systematic review. Am J Sports Med 2018;46(14): 3541-3549

5 Stoker AM, Stannard JP, Kuroki K, Bozynski CC, Pfeiffer FM, Cook JL. Validation of the Missouri Osteochondral Allograft Preservation System for the maintenance of osteochondral allograft quality during prolonged storage. Am J Sports Med 2018;46(01):58-65

6 Stannard JP, Cook JL. Prospective assessment of outcomes after primary unipolar, multisurface, and bipolar osteochondral allograft transplantations in the knee: a comparison of two preservation methods. Am J Sports Med 2020;48(06):1356-1364

7 Schreiner AJ, Stannard JP, Cook CR, et al. Comparison of meniscal allograft transplantation techniques using a preclinical canine model. J Orthop Res 2020; Epub ahead of print. Doi: 10.1002/ jor.24668

8 Rucinski K, Cook JL, Crecelius CR, Stucky R, Stannard JP. Effects of compliance with procedure-specific postoperative rehabilitation protocols on initial outcomes after osteochondral and meniscal allograft transplantation in the knee. Orthop J Sports Med 2019;7 (11):2325967119884291

9 Kuroki K, Stoker AM, Stannard JP, et al. Biologic joint repair strategies: the Mizzou BioJoint story. Toxicol Pathol 2017;45 (07):931-938

10 Sherman SL, Garrity J, Bauer K, Cook J, Stannard J, Bugbee W. Fresh osteochondral allograft transplantation for the knee: current concepts. J Am Acad Orthop Surg 2014;22(02):121-133

11 Cook JL, Stoker AM, Stannard JP, et al. A novel system improves preservation of osteochondral allografts. Clin Orthop Relat Res 2014;472(11):3404-3414

12 Baumann CA, Baumann JR, Bozynski CC, Stoker AM, Stannard JP, Cook JL. Comparison of techniques for preimplantation treatment of osteochondral allograft bone. J Knee Surg 2019;32(01):97-104

13 Stoker AM, Baumann CA, Stannard JP, Cook JL. Bone marrow aspirate concentrate versus platelet rich plasma to enhance osseous integration potential for osteochondral allografts. J Knee Surg 2018;31 (04):314-320
14 Hayden R, Rucinski K, Crecelius CR, Stucky R, Stannard JP, Cook JL. Outcomes associated with behavioral evaluation and counseling for patients undergoing orthopaedic surgery - a systematic review. J Orthop 2020;21:178-182

15 Melugin HP, Bernard CD, Camp CL, Saris DBF, Krych AJ. Bipolar cartilage lesions of the knee: a systematic review of techniques, outcomes, and complications. Cartilage 2019;•••:1947603519855761

16 Zouzias IC, Bugbee WD. Osteochondral allograft transplantation in the knee. Sports Med Arthrosc Rev 2016;24(02):79-84

17 Chui K, Jeys L, Snow M. Knee salvage procedures: the indications, techniques and outcomes of large osteochondral allografts. World J Orthop 2015;6(03):340-350

18 Crawford ZT, Schumaier AP, Glogovac G, Grawe BM. Return to sport and sports-specific outcomes after osteochondral allograft transplantation in the knee: a systematic review of studies with at least 2 years' mean follow-up. Arthroscopy 2019;35(06):1880-1889

19 Meric G, Gracitelli GC, Görtz S, De Young AJ, Bugbee WD. Fresh osteochondral allograft transplantation for bipolar reciprocal osteochondral lesions of the knee. Am J Sports Med 2015;43 (03):709-714

20 Beaver RJ, Mahomed M, Backstein D, Davis A, Zukor DJ, Gross AE. Fresh osteochondral allografts for post-traumatic defects in the knee. A survivorship analysis. J Bone Joint Surg Br 1992;74(01):105-110

21 Giannini S, Buda R, Ruffilli A, et al. Failures in bipolar fresh osteochondral allograft for the treatment of end-stage knee osteoarthritis. Knee Surg Sports Traumatol Arthrosc 2015;23 (07):2081-2089

22 Getgood A, Gelber J, Görtz S, De Young A, Bugbee W. Combined osteochondral allograft and meniscal allograft transplantation: a survivorship analysis. Knee Surg Sports Traumatol Arthrosc 2015; 23(04):946-953

23 Cotter EJ, Hannon CP, Christian DR, et al. Clinical outcomes of multifocal osteochondral allograft transplantation of the knee: An analysis of overlapping grafts and multifocal lesions. Am J Sports Med 2018;46(12):2884-2893

24 Krettek C, Clausen J, Omar M, Noack S, Neunaber C. Two-stage late reconstruction with a fresh large osteochondral shell allograft transplantation (FLOCSAT) for a large ostechondral defect in a non-union after a lateral tibia plateau fracture 2-year follow up. Injury 2017;48(07):1309-1318

25 Krettek C, Clausen JD, Bruns N, Neunaber C. Partial and complete joint transplantation with fresh osteochondral allografts-the FLOCSAT concept [in German]. Unfallchirurg 2017;120(11): 932-949

26 Krettek C, Clausen JD, Neunaber C. Complex joint reconstruction and joint transplantation with the FLOCSAT concept-planning and surgical implementation [in German]. Unfallchirurg 2017;120 (11):950-960

27 Assenmacher AT, Pareek A, Reardon PJ, Macalena JA, Stuart MJ, Krych AJ. Long-term outcomes after osteochondral allograft: a systematic review at long-term follow-up of 12.3 years. Arthroscopy 2016;32(10):2160-2168

28 Bayliss LE, Culliford D, Monk AP, et al. The effect of patient age at intervention on risk of implant revision after total replacement of the hip or knee: a population-based cohort study. Lancet 2017; 389(10077):1424-1430

29 Oladeji LO, Cook JL, Stannard JP, Crist BD. Large fresh osteochondral allografts for the hip: growing the evidence. Hip Int 2018;28 (03):284-290

30 Oladeji LO, Stannard JP, Cook CR, et al. Effects of autogenous bone marrow aspirate concentrate on radiographic integration of femoral condylar osteochondral allografts. Am J Sports Med 2017;45(12):2797-2803 